\title{
All maximal idempotent submonoids of $\mathrm{Hyp}_{\mathrm{G}}(2)$
}

\author{
Weerapong Wongpinit \\ Department of Mathematics, Faculty of \\ Science, Chiang Mai University, \\ Chiang Mai 50200, Thailand \\ email: wrpmathcmu@hotmail.com
}

\author{
Sorasak Leeratanavalee* \\ Department of Mathematics, Faculty of \\ Science, Chiang Mai University, \\ Chiang Mai 50200, Thailand \\ email: sorasak.1@cmu.ac.th
}

\begin{abstract}
The purpose of this paper is to determine all maximal idempotent submonoids and some maximal compatible idempotent submonoids of the monoid of all generalized hypersubstitutions of type $\tau=$ (2).
\end{abstract}

\section{Introduction}

In Universal Algebra, identities are used to classify algebras into collections, called varieties and hyperidentities are use to classify varieties into collections, called hypervarities. The concept of a hypersubstitution is a tool to study hyperidentities and hypervarities. The notion of a hypersubstitution originated by K. Denecke, D. Lau, R. Pöschel and D. Schweigert [3]. In 2000, S. Leeratanavalee and K. Denecke generalized the concepts of a hypersubstitution and a hyperidentity to the concepts of a generalized hypersubstitution and a strong hyperidentity, respectively [4]. The set of all generalized hypersubstitutions together with a binary operation and the identity hypersubstitution forms a monoid. There are several published papers on algebraic properties of this monoid and its submonoids.

2010 Mathematics Subject Classification: 20B30, 20M05, 20M17

Key words and phrases: generalized hypersubstitution, compatible, idempotent element, idempotent submonoids (*Corresponding author) 
The concept of regular subsemigroup plays an important role in the theory of semigroup. The concept of an idempotent submonoid is an example of a regular subsemigroup. In 2013, W. Puninagool and S. Leeratanavalee studied the natural partial order on the set $E\left(\mathrm{Hyp}_{\mathrm{G}}(2)\right)$ of all idempotent elements of $\mathrm{Hyp}_{\mathrm{G}}(2)$, see [6]. In 2012, the authors studied the natural partial order on $\mathrm{Hyp}_{\mathrm{G}}(2)$, see [7]. In this paper we determine all maximal idempotent submonoids and give some maximal compatible idempotent submonoids of $\mathrm{Hyp}_{\mathrm{G}}(2)$ under this partial order.

\section{Generalized hypersubstitutions}

Let $n \in \mathbb{N}$ be a natural number and $X_{n}:=\left\{x_{1}, x_{2}, \ldots, x_{n}\right\}$ be an $n$-element set. Let $\left\{f_{i} \mid i \in I\right\}$ be a set of $n_{i}$-ary operation symbols indexed by the set I. We call the sequence $\tau=\left(n_{i}\right)_{i \in I}$ of arities of $f_{i}$, the type. An $n$-ary term of type $\tau$ is defined inductively by the following.

(i) Every $x_{i} \in X_{n}$ is an $n$-ary term of type $\tau$.

(ii) If $t_{1}, t_{2}, \ldots, t_{n_{i}}$ are $n$-ary terms of type $\tau$, then $f_{i}\left(t_{1}, t_{2}, \ldots, t_{n_{i}}\right)$ is an $n$-ary term of type $\tau$.

We denote the smallest set which contains $x_{1}, \ldots, x_{n}$ and is closed under finite number of applications of (ii) by $W_{\tau}\left(X_{n}\right)$ and let $W_{\tau}(X):=\bigcup_{n=1}^{\infty} W_{\tau}\left(X_{n}\right)$ be the set of all terms of type $\tau$.

A mapping $\sigma$ from $\left\{f_{i} \mid i \in I\right\}$ into $W_{\tau}(X)$ which does not necessarily preserve the arity is called a generalized hypersubstitution of type $\tau$. The set of all generalized hypersubstitutions of type $\tau$ is denoted by $\mathrm{Hyp}_{\mathrm{G}}(\tau)$. In general, to combine two mappings together we use a composition of mappings. But in this case to combine two generalized hypersubstitutions we need the concept of a generalized superposition of terms and the extension of a generalized hypersubstitution which are defined by the following.

Definition 1 A generalized superposition of terms is a mapping $S^{m}: W_{\tau}(X)^{m+1} \longrightarrow W_{\tau}(X)$ where

(i) $S^{m}\left(x_{j}, t_{1}, \ldots, t_{m}\right):=t_{j}, 1 \leq j \leq m$,

(ii) $S^{m}\left(x_{j}, t_{1}, \ldots, t_{m}\right):=x_{j}, m<j \in \mathbb{N}$,

(iii) $S^{m}\left(f_{i}\left(s_{1}, \ldots, s_{n_{i}}\right), t_{1}, \ldots, t_{m}\right):=f_{i}\left(S^{m}\left(s_{1}, t_{1}, \ldots, t_{m}\right), \ldots\right.$, $\left.S^{m}\left(s_{n_{i}}, t_{1}, \ldots, t_{m}\right)\right)$. 
Definition 2 Let $\sigma \in \operatorname{Hyp}_{\mathrm{G}}(\tau)$. The extension of $\sigma$ is a mapping $\hat{\sigma}: W_{\tau}(X) \longrightarrow W_{\tau}(X)$ where

(i) $\hat{\sigma}[x]:=x \in X$,

(ii) $\hat{\sigma}\left[f_{i}\left(t_{1}, \ldots, t_{n_{i}}\right)\right]:=S^{n_{i}}\left(\sigma\left(f_{i}\right), \hat{\sigma}\left[t_{1}\right], \ldots, \hat{\sigma}\left[t_{n_{i}}\right]\right)$, for any $n_{i}$-ary operation symbol $f_{i}$ where $\hat{o}\left[t_{j}\right], 1 \leq j \leq n_{i}$ are already defined.

Proposition 1 ([4]) For arbitrary $t, t_{1}, t_{2}, \ldots, t_{n} \in W_{\tau}(X)$ and for arbitrary generalized hypersubstitution $\sigma, \sigma_{1}, \sigma_{2}$ we have

(i) $S^{\mathfrak{n}}\left(\hat{\sigma}[t], \hat{\sigma}\left[t_{1}\right], \ldots, \hat{\sigma}\left[t_{n}\right]\right)=\hat{\sigma}\left[S^{n}\left(t, t_{1}, \ldots, t_{n}\right)\right]$,

(ii) $\left(\hat{\sigma}_{1} \circ \sigma_{2}\right)^{\uparrow}=\hat{\sigma}_{1} \circ \hat{\sigma}_{2}$.

The binary operation of two generalized hypersubstitutions $\sigma_{1}, \sigma_{2}$ is defined by $\sigma_{1} \circ_{\mathrm{G}} \sigma_{2}:=\hat{\sigma}_{1} \circ \sigma_{2}$ where $\circ$ denotes the usual composition of mappings. It turns out that $\operatorname{Hyp}_{\mathrm{G}}(\tau)$ together with the identity element $\sigma_{i d}$ where $\sigma_{i d}\left(f_{i}\right)=$ $f_{i}\left(x_{1}, \ldots, x_{n_{i}}\right)$ is a monoid under $\circ_{G}$, see [4].

\section{All Maximal idempotent submonoids of $\mathrm{Hyp}_{\mathrm{G}}(2)$}

We recall first the definition of an idempotent element of a semigroup. Let $S$ be a semigroup. An element $a \in S$ is called idempotent if $a a=a$. We denote the set of all idempotent elements of a semigroup $S$ by $E(S)$. Let $E(S) \neq \emptyset$. Define $a \leq b(a, b \in E(S))$ iff $a=a b=b a$. Then $\leq$ is a partial order on $E(S)$. We call $\leq$ a natural partial order on $E(S)$. A natural partial order $\leq$ on a semigroup $S$ is said to be a compatible if $\mathrm{a} \leq \mathrm{b}$ implies $\mathrm{ac} \leq \mathrm{bc}$ and $\mathrm{ca} \leq \mathrm{cb}$ for all $a, b, c \in S$. Throughout this paper, let $f$ be a binary operation symbol of type $\tau=(2)$. By $\sigma_{t}$ we denote a generalized hypersubstitution which maps $f$ to the term $t \in W_{(2)}(X)$. For $t \in W_{(2)}(X)$ we introduce the following notation:

(i) leftmost $(t):=$ the first variable (from the left) occurring in $t$,

(ii) $\operatorname{rightmost}(\mathrm{t}):=$ the last variable occurring in $t$,

(iii) $\operatorname{var}(t):=$ the set of all variables occurring in $t$.

Let $\sigma_{\mathrm{t}} \in \operatorname{Hyp}_{\mathrm{G}}(2)$, we denote $R_{1}:=\left\{\sigma_{\mathrm{t}} \mid \mathrm{t}=\mathrm{f}\left(\mathrm{x}_{1}, \mathrm{t}^{\prime}\right)\right.$ where $\mathrm{t}^{\prime} \in \mathrm{W}_{(2)}(\mathrm{X})$ and $\left.x_{2} \notin \operatorname{var}\left(t^{\prime}\right)\right\}, R_{2}:=\left\{\sigma_{t} \mid t=f\left(t^{\prime}, x_{2}\right)\right.$ where $t^{\prime} \in W_{(2)}(X)$ and $x_{1} \notin$ $\left.\operatorname{var}\left(t^{\prime}\right)\right\}, R_{3}:=\left\{\sigma_{t} \mid t \in\left\{x_{1}, x_{2}, f\left(x_{1}, x_{2}\right)\right\}\right\}$ and $R_{4}:=\left\{\sigma_{t} \mid \operatorname{var}(t) \cap\left\{x_{1}, x_{2}\right\}=\emptyset\right\}$. 
In 2008, W. Puninagool and S. Leeratanavalee [5] proved that: $\bigcup_{i=1}^{4} R_{i}=$ $E\left(\operatorname{Hyp}_{G}(2)\right)$.

Example 1 Let $\sigma_{s} \in R_{1}$ and $\sigma_{t} \in R_{2}$ such that $s=f\left(x_{1}, s^{\prime}\right)$ and $t=f\left(t^{\prime}, x_{2}\right)$ where $s^{\prime}=f\left(x_{4}, x_{1}\right)$ and $t^{\prime}=f\left(x_{2}, x_{6}\right)$. Consider

$$
\begin{aligned}
\left(\sigma_{s} \circ_{G} \sigma_{t}\right)(f) & =\widehat{\sigma}_{s}\left[f\left(f\left(x_{2}, x_{6}\right), x_{2}\right)\right] \\
& =S^{2}\left(f\left(x_{1}, f\left(x_{4}, x_{1}\right)\right), \widehat{\sigma}_{s}\left[f\left(x_{2}, x_{6}\right)\right], \widehat{\sigma}_{s}\left[x_{2}\right]\right) \\
& =S^{2}\left(f\left(x_{1}, f\left(x_{4}, x_{1}\right), f\left(x_{2}, f\left(x_{4}, x_{2}\right)\right), x_{2}\right)\right. \\
& =f\left(f\left(x_{2}, f\left(x_{4}, x_{2}\right)\right), f\left(x_{4}, f\left(x_{2}, f\left(x_{4}, x_{2}\right)\right)\right)\right) .
\end{aligned}
$$

So $\sigma_{\mathrm{s}} \circ_{\mathrm{G}} \sigma_{\mathrm{t}} \notin \bigcup_{i=1}^{4} R_{i}$.

By the previous example, we have $\bigcup_{i=1}^{4} R_{i}$ is not a subsemigroup of Hyp $p_{G}$ (2).

Let $\sigma_{\mathrm{t}} \in \operatorname{Hyp}_{\mathrm{G}}(2)$, we denote $R_{1}^{\prime}:=\left\{\sigma_{\mathrm{t}} \mid \mathrm{t}=\mathrm{f}\left(\mathrm{x}_{1}, \mathrm{t}^{\prime}\right)\right.$ where $\mathrm{t}^{\prime} \in \mathrm{W}_{(2)}(\mathrm{X}), \mathrm{x}_{2} \notin$ $\operatorname{var}\left(t^{\prime}\right)$ and rightmost $\left.\left(t^{\prime}\right) \neq x_{1}\right\}$ and $R_{2}^{\prime}:=\left\{\sigma_{t} \mid t=f\left(t^{\prime}, x_{2}\right)\right.$ where $t^{\prime} \in$ $W_{(2)}(X), x_{1} \notin \operatorname{var}\left(t^{\prime}\right)$ and leftmost $\left.\left(t^{\prime}\right) \neq x_{2}\right\}$.

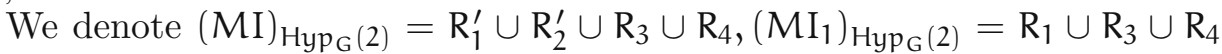
and $\left(M_{2}\right)_{H_{y p}(2)}=R_{2} \cup R_{3} \cup R_{4}$.

Proposition $2(\mathrm{MI})_{\mathrm{Hyp}_{\mathrm{G}}(2)}$ is an idempotent submonoid of $\mathrm{Hyp} \mathrm{G}_{\mathrm{G}}(2)$.

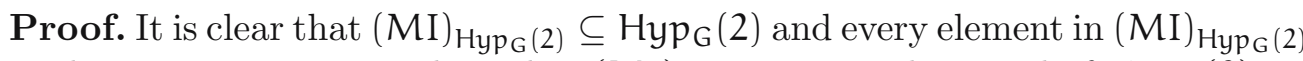
is idempotent. Next, we show that $(M I)_{H_{y p}(2)}$ is a submonoid of $\operatorname{Hyp}_{\mathrm{G}}(2)$.

Case 1: $\sigma_{t} \in R_{1}^{\prime}$. Then $t=f\left(x_{1}, t^{\prime}\right)$ where $t^{\prime} \in W_{(2)}(X)$ such that $x_{2} \notin \operatorname{var}\left(t^{\prime}\right)$ and rightmost $\left(t^{\prime}\right) \neq x_{1}$. Let $\sigma_{s} \in(M I)_{H_{y p}(2)}$.

Case 1.1: $\sigma_{s} \in R_{1}^{\prime}$. Then $s=f\left(x_{1}, s^{\prime}\right)$ where $x_{2} \notin \operatorname{var}\left(s^{\prime}\right)$ and $\operatorname{rightmost}\left(s^{\prime}\right) \neq x_{1}$. Consider

$$
\begin{aligned}
\left(\sigma_{\mathrm{t}} \circ_{\mathrm{G}} \sigma_{\mathrm{s}}\right)(\mathrm{f}) & =\widehat{\sigma}_{\mathrm{t}}\left[\mathrm{f}\left(\mathrm{x}_{1}, \mathrm{~s}^{\prime}\right)\right] \\
& =\mathrm{S}^{2}\left(\mathrm{f}\left(\mathrm{x}_{1}, \mathrm{t}^{\prime}\right), \mathrm{x}_{1}, \widehat{\sigma}_{\mathrm{t}}\left[\mathrm{s}^{\prime}\right]\right) \\
& =\mathrm{f}\left(\mathrm{x}_{1}, \mathrm{t}^{\prime}\right) \quad \text { since } x_{2} \notin \operatorname{var}\left(\mathrm{t}^{\prime}\right) .
\end{aligned}
$$

Then $\sigma_{\mathrm{t}} \circ_{\mathrm{G}} \sigma_{\mathrm{s}} \in \mathrm{R}_{1}^{\prime} \subseteq(\mathrm{MI})_{\mathrm{Hyp}_{\mathrm{G}}(2)}$.

Case 1.2: $\sigma_{s} \in R_{2}^{\prime}$. Then $s=f\left(s^{\prime}, x_{2}\right)$ where $x_{1} \notin \operatorname{var}\left(s^{\prime}\right)$ and leftmost $\left(s^{\prime}\right) \neq x_{2}$. Consider $\left(\sigma_{s} \circ_{G} \sigma_{t}\right)(f)=\widehat{\sigma}_{s}\left[f\left(x_{1}, t^{\prime}\right)\right]=S^{2}\left(f\left(s^{\prime}, x_{2}\right), x_{1}, \widehat{\sigma}_{s}\left[t^{\prime}\right]\right)$ 
$=f\left(S^{2}\left(s^{\prime}, x_{1}, w\right), S^{2}\left(x_{2}, x_{1}, w\right)\right)$, where $w=\widehat{\sigma}_{s}\left[t^{\prime}\right]$. Since $x_{2} \notin v \operatorname{var}\left(t^{\prime}\right)$ and $\operatorname{rightmost}\left(t^{\prime}\right) \neq x_{1}$, then $x_{1}, x_{2} \notin \operatorname{var}(w)$. Since $x_{1} \notin \operatorname{var}\left(s^{\prime}\right)$ and $x_{1}, x_{2} \notin$ $\operatorname{var}(w)$, then $x_{1}, x_{2} \notin \operatorname{var}\left(S^{2}\left(s^{\prime}, x_{1}, w\right)\right)$. Consider $\left(\sigma_{t} \circ_{G} \sigma_{s}\right)(f)=\widehat{\sigma}_{t}\left[f\left(s^{\prime}, x_{2}\right)\right]=$ $S^{2}\left(f\left(x_{1}, t^{\prime}\right), \widehat{\sigma}_{t}\left[s^{\prime}\right], x_{2}\right)=f\left(S^{2}\left(x_{1}, u, x_{2}\right), S^{2}\left(t^{\prime}, u, x_{2},\right)\right)$, where $u=\widehat{\sigma}_{t}\left[s^{\prime}\right]$. Since $x_{1} \notin \operatorname{var}\left(s^{\prime}\right)$ and leftmost $\left(s^{\prime}\right) \neq x_{2}$, we have $x_{1}, x_{2} \notin \operatorname{var}(u)$. Since $x_{2} \notin$ $\operatorname{var}\left(t^{\prime}\right)$ and $x_{1}, x_{2} \notin \operatorname{var}(u)$, we have $x_{1}, x_{2} \notin \operatorname{var}\left(S^{2}\left(t^{\prime}, u, x_{2}\right)\right)$. Then $\sigma_{s} \circ_{G}$ $\sigma_{\mathrm{t}}, \sigma_{\mathrm{t}} \circ_{\mathrm{G}} \sigma_{\mathrm{s}} \in \mathrm{R}_{4}^{\prime} \subseteq(\mathrm{MI})_{\mathrm{Hyp}_{\mathrm{G}}(2)}$.

Case 1.3: $\sigma_{s} \in R_{3}$. Then $s=x_{1}$ or $s=x_{2}$ or $s=f\left(x_{1}, x_{2}\right)$.

If $s=x_{1}$, then $\left(\sigma_{t} \circ_{G} \sigma_{s}\right)(f)=\widehat{\sigma}_{t}\left[x_{1}\right]=x_{1}$ and $\left(\sigma_{s} \circ_{G} \sigma_{t}\right)(f)=\widehat{\sigma}_{x_{1}}\left[f\left(x_{2}, t^{\prime}\right)\right]=$ $S^{2}\left(x_{1}, x_{2}, \widehat{\sigma}_{x_{1}}\left[t^{\prime}\right]\right)=x_{2}$.

If $s=x_{2}$, then $\left(\sigma_{t} \circ_{G} \sigma_{s}\right)(f)=\widehat{\sigma}_{t}\left[x_{2}\right]=x_{2}$ and $\left(\sigma_{s} \circ_{G} \sigma_{t}\right)(f)=\widehat{\sigma}_{x_{2}}\left[f\left(x_{2}, t^{\prime}\right)\right]=$ $S^{2}\left(x_{2}, x_{2}, \widehat{\sigma}_{x_{2}}\left[t^{\prime}\right]\right)$.

Since $x_{1} \notin \operatorname{var}\left(\mathrm{t}^{\prime}\right)$ and rightmost $\left(\mathrm{t}^{\prime}\right) \neq x_{2}$, then $S^{2}\left(x_{2}, x_{2}, \widehat{\sigma}_{x_{2}}\left[t^{\prime}\right]\right)=x_{i} \notin$ $\left\{x_{1}, x_{2}\right\}$.

If $s=f\left(x_{1}, x_{2}\right)$, then $\sigma_{s}=\sigma_{i d}$ such that $\sigma_{t} \circ_{G} \sigma_{i d}=\sigma_{t}=\sigma_{i d} \circ_{G} \sigma_{t}$.

Therefore $\sigma_{s} \circ_{G} \sigma_{t}, \sigma_{s} \circ_{G} \sigma_{t} \in(M I)_{H_{y p}(2)}$.

Case 1.4: $\sigma_{s} \in R_{4}$. Then $s=f\left(s_{1}, s_{2}\right)$ where $x_{1}, x_{2} \notin \operatorname{var}(s)$. Consider $\left(\sigma_{t} \circ_{G} \sigma_{s}\right)(f)=\widehat{\sigma}_{t}\left[f\left(s_{1}, s_{2}\right)\right]=S^{2}\left(f\left(x_{2}, t^{\prime}\right), \widehat{\sigma}_{t}\left[s_{1}\right], \widehat{\sigma}_{t}\left[s_{2}\right]\right)=f\left(S^{2}\left(x_{2}, w_{1}, w_{2}\right)\right.$, $\left.S^{2}\left(t^{\prime}, w_{1}, w_{2}\right)\right)$, where $w_{1}=\widehat{\sigma}_{t}\left[s_{1}\right]$ and $w_{2}=\widehat{\sigma}_{t}\left[s_{2}\right]$. Then $x_{1}, x_{2} \notin \operatorname{var}\left(w_{1}\right) \cup$ $\operatorname{var}\left(w_{2}\right)$. The consequence is $x_{1}, x_{2} \notin \operatorname{var}\left(S^{2}\left(t^{\prime}, w_{1}, w_{2}\right)\right)$.

Since $x_{1}, x_{2} \notin \operatorname{var}\left(w_{2}\right) \cup \operatorname{var}\left(S^{2}\left(t^{\prime}, w_{1}, w_{2}\right)\right)$, so that $\sigma_{t} \circ_{G} \sigma_{s} \in R_{4}^{\prime} \subseteq(M I)_{H_{y p}(2)}$. Consider $\left(\sigma_{s} \circ_{G} \sigma_{t}\right)(f)=\widehat{\sigma}_{s}\left[f\left(x_{2}, t^{\prime}\right)\right]=S^{2}\left(f\left(s_{1}, s_{2}\right), x_{2}, \widehat{\sigma}_{s}\left[t^{\prime}\right]\right)=f\left(s_{1}, s_{2}\right)$ since $x_{1}, x_{2} \notin \operatorname{var}(s)$. So that $\sigma_{s} \circ_{G} \sigma_{t} \in R_{4} \subseteq(M I)_{H_{y p}(2)}$.

Case 2: $\sigma_{t} \in R_{2}^{\prime}$ and $\sigma_{s} \in R_{2}^{\prime} \cup R_{3} \cup R_{4}$. It can be proved similarly as in Case 1. Then we have $\sigma_{t} \circ_{G} \sigma_{s}, \sigma_{s} \circ_{G} \sigma_{t} \in(M I)_{H y p}(2)$.

Case 3: $\sigma_{t} \in R_{3}$ and $\sigma_{s} \in R_{3} \cup R_{4}$. It can be proved similarly as in Case 1.3. Then we have $\sigma_{t} \circ_{G} \sigma_{s}, \sigma_{s} \circ_{G} \sigma_{t} \in(M I)_{H_{y p}(2)}$.

Case 4: $\sigma_{t} \in R_{4}$ and $\sigma_{s} \in R_{4}$. Then $\sigma_{t} \circ_{G} \sigma_{s}=\sigma_{t} \in R_{4} \subseteq(M I)_{H_{y p}(2)}$.

Therefore $(M I)_{H_{y p}(2)}$ is a submonoid of $\mathrm{Hyp}_{\mathrm{G}}(2)$.

Corollary $1\left(\mathrm{MI}_{1}\right)_{\mathrm{Hyp}_{\mathrm{G}}(2)}$ and $\left(\mathrm{MI}_{2}\right)_{\mathrm{Hyp}_{\mathrm{G}}(2)}$ are idempotent submonoids of $\operatorname{Hyp}_{\mathrm{G}}(2)$.

Proposition $3(\mathrm{MI})_{\mathrm{Hyp}_{\mathrm{G}}(2)}$ is a maximal idempotent submonoid of $\mathrm{Hyp}_{\mathrm{G}}(2)$.

Proof. Let $K$ be a proper idempotent submonoid of $\mathrm{Hyp}_{\mathrm{G}}(2)$ such that

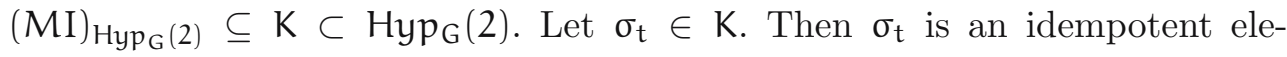
ment.

Case 1: $\sigma_{t} \in R_{1} \backslash R_{1}^{\prime}$. Then $t=f\left(x_{1}, t^{\prime}\right)$ where $x_{2} \notin \operatorname{var}\left(t^{\prime}\right)$ and $\operatorname{rightmost}\left(t^{\prime}\right)=$ 
$x_{1}$. Choose $\sigma_{s} \in R_{2}^{\prime} \subseteq K$, then $s=f\left(s^{\prime}, x_{2}\right)$ such that $x_{1} \notin \operatorname{var}\left(s^{\prime}\right)$ and leftmost $\left(s^{\prime}\right) \neq x_{2}$. Consider $\left(\sigma_{s} \circ{ }_{G} \sigma_{t}\right)(f)=\widehat{\sigma}_{s}\left[f\left(x_{1}, t^{\prime}\right)\right]=S^{2}\left(f\left(s^{\prime}, x_{2}\right), x_{1}, \widehat{\sigma}_{s}\left[t^{\prime}\right]\right)$ $=f\left(S^{2}\left(s^{\prime}, x_{1}, w\right), S^{2}\left(x_{2}, x_{1}, w\right)\right)$ where $w=\widehat{\sigma}_{s}\left[t^{\prime}\right]$. Since $x_{2} \in \operatorname{var}(s)$ and rightmost $\left(t^{\prime}\right)=x_{1}$, we have $x_{1} \in \operatorname{var}(w)$ and $S^{2}\left(s^{\prime}, x_{1}, w\right) \in W_{(2)}(X) \backslash X$. Since $x_{1} \in \operatorname{var}(w), \sigma_{s} \circ_{G} \sigma_{t}$ is not idempotent. So $\sigma_{t} \in R_{1}^{\prime}$.

Case 2: $\sigma_{t} \in R_{2} \backslash R_{2}^{\prime}$. Then $t=f\left(t^{\prime}, x_{2}\right)$ where $x_{1} \notin \operatorname{var}\left(t^{\prime}\right)$ and leftmost $\left(t^{\prime}\right)=$ $x_{2}$. Choose $\sigma_{s} \in R_{1}^{\prime} \subseteq K$, then $s=f\left(x_{1}, s^{\prime}\right)$ such that $x_{2} \notin \operatorname{var}\left(s^{\prime}\right)$ and rightmost $\left(s^{\prime}\right) \neq x_{1}$. Consider $\left(\sigma_{s} \circ_{G} \sigma_{t}\right)(f)=\widehat{\sigma}_{s}\left[f\left(t^{\prime}, x_{2}\right)\right]=S^{2}\left(f\left(x_{1}, s^{\prime}\right), \widehat{\sigma}_{s}\left[t^{\prime}\right]\right.$, $\left.x_{2}\right)=f\left(S^{2}\left(x_{1}, w, x_{2}\right), S^{2}\left(s^{\prime}, w, x_{2}\right)\right)$, where $w=\widehat{\sigma}_{s}\left[t^{\prime}\right]$. Since $x_{1} \in \operatorname{var}(s)$ and leftmost $\left(t^{\prime}\right)=x_{2}$, we have $x_{2} \in \operatorname{var}(w)$ and $S^{2}\left(s^{\prime}, w, x_{2}\right) \in W_{(2)}(X) \backslash X$. Since $x_{2} \in \operatorname{var}(w), \sigma_{s} \circ_{G} \sigma_{t}$ is not idempotent. So $\sigma_{t} \in R_{2}^{\prime}$. Then $\sigma_{t} \in(M I)_{H_{y p}(2)}$. Therefore $\mathrm{K} \subseteq(\mathrm{MI})_{\mathrm{Hyp}_{\mathrm{G}}(2)}$ and thus $\mathrm{K}=(\mathrm{MI})_{\mathrm{Hyp}_{\mathrm{G}}(2)}$.

Proposition $4\left(\mathrm{MI}_{1}\right)_{\mathrm{Hyp}_{\mathrm{G}}(2)}$ is a maximal idempotent submonoid of $\mathrm{Hyp}_{\mathrm{G}}(2)$.

Proof. Let $K$ be a proper idempotent submonoid of $\mathrm{Hyp}_{\mathrm{G}}(2)$ such that $\left(\mathrm{MI}_{1}\right)_{\mathrm{Hyp}_{\mathrm{G}}(2)} \subseteq \mathrm{K} \subset \mathrm{Hyp}_{\mathrm{G}}(2)$. Let $\sigma_{\mathrm{t}} \in \mathrm{K}$. Then $\sigma_{\mathrm{t}}$ is an idempotent element. If $\sigma_{t} \in R_{2}$. Then $t=f\left(t^{\prime}, x_{2}\right)$ where $x_{1} \notin \operatorname{var}\left(t^{\prime}\right)$. Choose $\sigma_{s} \in R_{1}$ such that $s=f\left(x_{1}, s^{\prime}\right)$ where $x_{2} \notin \operatorname{var}\left(s^{\prime}\right), s^{\prime} \in W_{(2)}(X) \backslash X$ and $\operatorname{rightmost}\left(s^{\prime}\right)=x_{1}$. Consider $\left(\sigma_{t} \circ_{G} \sigma_{s}\right)(f)=\widehat{\sigma}_{t}\left[f\left(x_{1}, s^{\prime}\right)\right]=S^{2}\left(f\left(t^{\prime}, x_{2}\right), x_{1}, \widehat{\sigma}_{t}\left[s^{\prime}\right]\right)=f\left(S^{2}\left(t^{\prime}, x_{1}, w\right)\right.$, $\left.S^{2}\left(x_{2}, x_{1}, w\right)\right)$, where $w=\widehat{\sigma}_{t}\left[s^{\prime}\right]$. Since $x_{2} \in \operatorname{var}(t)$, we have $x_{1} \in \operatorname{var}(w)$ and $S^{2}\left(t^{\prime}, x_{1}, w\right) \in W_{(2)}(X) \backslash X$. Since $x_{1} \in \operatorname{var}(w), \sigma_{t} o_{G} \sigma_{s}$ is not idempotent, so $\sigma_{t} \in\left(M_{1}\right)_{\mathrm{Hyp}_{\mathrm{G}}(2)}$. Therefore $\mathrm{K}=\left(\mathrm{MI}_{1}\right)_{\mathrm{Hyp}_{\mathrm{G}}(2)}$.

Proposition $5\left(\mathrm{MI}_{2}\right)_{\mathrm{Hyp}_{\mathrm{G}}(2)}$ is a maximal idempotent submonoid of $\mathrm{Hyp}_{\mathrm{G}}(2)$.

Proof. The proof is similar to the proof of Proposition 4.

Corollary $2\left\{(\mathrm{MI})_{\mathrm{Hyp}_{\mathrm{G}}(2)},\left(\mathrm{MI}_{1}\right)_{\mathrm{Hyp}_{\mathrm{G}}(2)},\left(\mathrm{MI}_{2}\right)_{\mathrm{Hyp}_{\mathrm{G}}(2)}\right\}$ is the set of all maximal idempotent submonoids of $\mathrm{Hyp}_{\mathrm{G}}(2)$.

Proposition $6([6])$ Let $\sigma_{\mathrm{t}}$ be an idempotent element. Then $\sigma_{x_{1}} \leq \sigma_{\mathrm{t}}$ if and only if leftmost $(\mathrm{t})=\mathrm{x}_{1}$.

Proposition 7 ([6]) Let $\sigma_{\mathrm{t}}$ be an idempotent element. Then $\sigma_{x_{2}} \leq \sigma_{\mathrm{t}}$ if and only if rightmost $(\mathrm{t})=\mathrm{x}_{2}$.

Proposition 8 For each $\mathrm{t} \in \mathrm{W}_{(2)}(\mathrm{X})$ where $\mathrm{x}_{2} \notin \operatorname{var}(\mathrm{t}),\left\{\sigma_{\mathrm{x}_{1}}, \sigma_{\mathrm{id}}, \sigma_{\mathrm{f}\left(\mathrm{x}_{1}, \mathrm{t}\right)}\right\}$ is a maximal compatible idempotent submonoid of $\mathrm{Hyp}_{\mathrm{G}}(2)$. 
Proof. By using Proposition 6, $\sigma_{x_{1}} \leq \sigma_{f\left(x_{1}, t\right)}$. Then $\sigma_{x_{1}}=\sigma_{x_{1}} \circ_{G} \sigma_{f\left(x_{1}, t\right)}=$ $\sigma_{f\left(x_{1}, t\right)} \circ_{G} \sigma_{x_{1}}$ and $\sigma_{i d}$ is the identity element. We have $\left\{\sigma_{x_{1}}, \sigma_{i d}, \sigma_{f\left(x_{1}, t\right)}\right\}$ is an idempotent submonoid of $\mathrm{Hyp}_{\mathrm{G}}(2)$. Since

$\sigma_{f\left(x_{1}, t\right)} \circ_{G} \sigma_{x_{1}}=\sigma_{x_{1}} \circ_{G} \sigma_{f\left(x_{1}, t\right)}=\sigma_{x_{1}} \circ_{G} \sigma_{x_{1}}=\sigma_{x_{1}} \leq \sigma_{f\left(x_{1}, t\right)}=\sigma_{f\left(x_{1}, t\right)} \circ_{G} \sigma_{f\left(x_{1}, t\right)}$.

We have $\left\{\sigma_{x_{1}}, \sigma_{i d}, \sigma_{f\left(x_{1}, t\right)}\right\}$ is a compatible idempotent submonoid of Hyp $p_{G}(2)$.

Let $\mathrm{K}$ be a proper compatible idempotent submonoid of $\mathrm{Hyp}_{\mathrm{G}}(2)$ such that $\left\{\sigma_{x_{1}}, \sigma_{i d}, \sigma_{f\left(x_{1}, t\right)}\right\} \subseteq \mathrm{K} \subset \operatorname{Hyp}_{\mathrm{G}}(2)$. Let $\sigma_{\mathrm{s}} \in \mathrm{K}$. Then $\sigma_{\mathrm{s}}$ is an idempotent element.

Case 1: $\sigma_{s} \in R_{1} \backslash\left\{\sigma_{x_{1}}, \sigma_{i d}, \sigma_{f\left(x_{1}, t\right)}\right\}$. Then $s=f\left(x_{1}, s^{\prime}\right)$ where $x_{2} \notin \operatorname{var}\left(s^{\prime}\right)$. Since $K$ is a compatible idempotent submonoid and $\sigma_{f\left(x_{1}, t\right)} \leq \sigma_{i d}$, we have $\sigma_{\mathrm{f}\left(\mathrm{x}_{1}, \mathrm{t}\right)} \circ_{\mathrm{G}} \sigma_{\mathrm{f}\left(\mathrm{x}_{1}, \mathrm{~s}^{\prime}\right)}=\sigma_{\mathrm{f}\left(\mathrm{x}_{1}, \mathrm{t}\right)} \leq \sigma_{\mathrm{f}\left(\mathrm{x}_{1}, \mathrm{~s}^{\prime}\right)}=\sigma_{\mathrm{id}} \circ_{\mathrm{G}} \sigma_{\mathrm{f}\left(\mathrm{x}_{1}, \mathrm{~s}^{\prime}\right)}$ which is a contradiction.

Case 2: $\sigma_{s} \in R_{2}$. Then $s=f\left(s^{\prime}, x_{2}\right)$ where $x_{1} \notin \operatorname{var}\left(s^{\prime}\right)$. Since $K$ is a compatible idempotent submonoid and $\sigma_{\mathrm{f}\left(s^{\prime}, x_{2}\right)} \leq \sigma_{\mathrm{id}}$, we have $\sigma_{\mathrm{x}_{1}} \circ_{\mathrm{G}} \sigma_{\mathrm{f}\left(s^{\prime}, \mathrm{x}_{2}\right)}=$ $\sigma_{\text {leftmost }\left(s^{\prime}\right)} \leq \sigma_{x_{1}}=\sigma_{x_{1}} \circ_{G} \sigma_{f\left(x_{1}, s^{\prime}\right)}$. So leftmost $\left(s^{\prime}\right)=x_{1}$ which is a contradiction.

Case 3: $\sigma_{s}=\sigma_{x_{2}}$. Since $K$ is a compatible idempotent submonoid and $\sigma_{x_{1}} \leq$ $\sigma_{i d}$, we have $\sigma_{x_{2}} \circ_{G} \sigma_{x_{1}}=\sigma_{x_{1}} \leq \sigma_{x_{2}}=\sigma_{x_{2}} \circ_{G} \sigma_{i d}$ which is a contradiction.

Case 4: $\sigma_{s} \in R_{4}$. Then $s=f\left(s_{1}, s_{2}\right) \in W_{(2)} X \backslash X$ where $x_{1}, x_{2} \notin \operatorname{var}(s)$. Since $K$ is a compatible idempotent submonoid and $\sigma_{x_{1}} \leq \sigma_{i d}$, we have $\sigma_{\mathrm{s}} \circ_{\mathrm{G}} \sigma_{\chi_{1}}=\sigma_{x_{1}} \leq \sigma_{\mathrm{s}}=\sigma_{\mathrm{s}} \circ_{\mathrm{G}} \sigma_{i d}$ which is a contradiction.

Therefore $K=\left\{\sigma_{x_{1}}, \sigma_{i d}, \sigma_{f\left(x_{1}, t\right)}\right\}$ is a maximal compatible idempotent submonoid of $\mathrm{Hyp}_{\mathrm{G}}(2)$.

Proposition 9 For each $\mathrm{t} \in \mathrm{W}_{(2)} \mathrm{X}$ where $\mathrm{x}_{1} \notin \operatorname{var}(\mathrm{t}),\left\{\sigma_{\mathrm{x}_{2}}, \sigma_{\mathrm{id}}, \sigma_{\mathrm{f}\left(\mathrm{t}, \mathrm{x}_{2}\right)}\right\}$ is a maximal compatible idempotent submonoid of $\mathrm{Hyp}_{\mathrm{G}}(2)$.

Proof. The proof is similar to the proof of Proposition 8.

\section{Acknowledgements}

This research was supported by the Human Resource Development in Science Project (Science Achievement Scholarship of Thailand, SAST) and the Graduate school, Chiang Mai University, Chiang Mai 50200, Thailand. The corresponding author was supported by Faculty of Science, Chiang Mai University, Chiang Mai 50200, Thailand. 


\section{References}

[1] A. Boonmee, S. Leeratanavalee, Factorisable Monoid of Generalized Hypersubstitutions of Type $\tau=(2)$, to appear in Thai Journal of Mathematics, (2014).

[2] J.M. Howie, Fundamentals of Semigroup Theory, Academic Press, London, 1995.

[3] K. Denecke, D. Lau, R. Pöschel, D. Schweigert, Hyperidentities, hyperequational classes and clone congruences, Contributions to General Algebra, 7, Verlag Hölder-Pichler-Tempsky, Wien (1991), 97-118.

[4] S. Leeratanavalee, K. Denecke, Generalized Hypersubstitutions and Strongly Solid Varieties, General Algebra and Applications, Proc. of the "59 th Workshop on General Algebra", "15 th Conference for Young Algebraists Potsdam 2000", Shaker Verlag (2000), 135-145.

[5] W. Puninagool, S. Leeratanavalee, The Order of Generalized Hypersubstitutions of Type $\tau=(2)$, International Journal of Mathematics and Mathematical Sciences, 2008 (2008), Article ID 263541, 8 pages, doi:10.1155/2008/263541.

[6] W. Puningool, S. Leeratanavalee, Natural Partial Ordering on $\mathrm{E}\left(\mathrm{Hyp}_{\mathrm{G}}(2)\right)$, Asian-European Journal of Mathematics, 6 (2) (2013), 1350016, 7 pages, doi:10.1142/S1793557113500162.

[7] W. Wongpinit, S. Leeratanavalee, Natural Partial Order on Hyp $\mathrm{G}_{\mathrm{G}}(2)$, EastWest Journal of Mathematics, 14(1) (2012), 54-66. 